\title{
Parallel and Perpendicular Lamellae on Corrugated Surfaces
}

\author{
Yoav Tsori \\ Laboratoire Matière Molle \& Chimie (UMR 167) \\ ESPCI, 10 rue Vauquelin, 75231 Paris CEDEX 05, France \\ David Andelman \\ School of Physics and Astronomy \\ Raymond and Beverly Sackler Faculty of Exact Sciences \\ Tel Aviv University, Tel Aviv 69978, Israel
}

$15 / 4 / 2003$

\begin{abstract}
We consider the relative stability of parallel and perpendicular lamellar layers on corrugated surfaces. The model can be applied to smectic phases of liquid crystals, to lamellar phases of shortchain amphiphiles and to lamellar phases of long-chain block copolymers. The corrugated surface is modelled by having a single $q$-mode lateral corrugation of a certain height. The lamellae deform close to the surface as a result of chemical interaction with it. The competition between the energetic cost of elastic deformations and the gain in surface energy determines whether parallel or perpendicular lamellar orientation (with respect to the surface) is preferred. Our main results are summarized in two phase diagrams, each exhibiting a transition line from the parallel to perpendicular orientations. The phase diagrams depend on the three system parameters: the lamellar natural periodicity, and the periodicity and amplitude of surface corrugations. For a fixed lamellar periodicity (or polymer chain length), the parallel orientation is preferred as the amplitude of surface corrugation decreases and/or its periodicity increases. Namely, for surfaces having small corrugations centered at long wavelengths. For a fixed corrugation periodicity, the parallel orientation is preferred for small corrugation amplitude and/or large lamellae periodicity. Our results are in agreement with recent experimental results carried out on thin block copolymer films of PS-PMMA (polystyrene-polymethylmethacrylate) in the lamellar phase, and in contact with several corrugated surfaces.
\end{abstract}




\section{Introduction}

Numerous ways to control the orientation of ordered meso-phases have been extensively studied in recent years. As an example, we mention that lamellar block copolymers (BCP) confined between two flat and parallel surfaces have been shown to orient parallel or perpendicular to the surfaces, depending on the surface separation 1, 2]. Furthermore, chemically patterned surface can induce perpendicular or even tilted lamellae, if the chemical interaction is strong enough [3] 4. Applying shear is yet another effective method in producing large well-aligned samples [5], but this method is difficult to implement in thin films. Another related situation can be found in liquid-crystals, where surface anchoring determines the direction of adjacent molecules and affects the bulk orientation and possible defects [7]. This type of surface effect has been studied extensively in relation to the Fréedericksz transition and in twisted nematic liquid-crystal displays. Electric field can be very useful in aligning samples in which a large dielectric or conductivity contrast exists between the components [8, 9, 10, 11, 12, 13, 14, 15, 16. However, the use of electric field requires rather sophisticated experimental setups in order to avoid adverse effects of ion accumulation at electrodes, short-circuits due to dust particles, etc. 16.

Most of the studies mentioned above are restricted to films in contact with flat and smooth solid surfaces. Little attention has been paid to the role of surface roughness on film morphology and orientation, although it is clear that non-flat surface topography gives rise to defects (e.g. vacancies in the lamellar ordering) and affects the lamellae orientation. Quite recently, experiments carried out by Hashimoto and co-workers on lamellar BCP films [17. addressed the question of how surface roughness affects BCP film orientation. In their study it was shown that the degree of surface roughness controls the lamellar orientation, leading to situations where the BCP films orient themselves parallel or perpendicular to the surface. These experiments serve as a starting point of our theoretical investigation, where we restrict ourselves to lamellar multilayer systems in contact with one corrugated surface. The model is expressed in terms of the elastic energy of lamellar or smectic systems, and is described by only two elastic constants. Hence, although some system-specific details are missing, the results are not restricted to BCP films, but are more general and equally apply to a broad class of systems ranging from smectic liquid-crystals to lyotropic (oil/water/amphiphile) systems. The main difference between these systems is in the values of the system parameters: the lamellar periodicity, strength of surface interaction and elastic constants.

The elastic deformation energy of lamellar layers is studied separately for parallel and perpendicular orientations. It is shown that depending on the surface corrugation amplitude and periodicity, phase transitions can occur between the two orientations. Since real surfaces are never ideally flat, understanding and characterizing surface roughness can be of great importance in controlling orientation of lamellar phases. This orientation mechanism is complementary to the mechanisms mentioned above of electric fields, shear and chemical surface patterning. In Sections 2 and 3 we calculate the deviations of lamellae from their corresponding flat perpendicular and parallel states. The free energy of the parallel and perpendicular states are compared in Sec. [ and a general discussion and comparison with previous works follows in Sec. [5

\section{Perpendicular Layers on Corrugated Surface}

Consider a lamellar system confined by one topographically corrugated surface, as depicted in Fig. 1. Instead of considering a rough surface having a random and quenched topography, we assume that the surface is characterized by a typical corrugation with a single amplitude and wavelength. The difference between a true rough surface and a corrugated one is not expected to be very significant as long as the rough surface amplitude and wavelengths do not vary much about their average values. A further 
simplification is that the surface height $h$ (measured along the $z$-direction) is taken to depend only on the lateral $x$ direction, while it is translational invariant in the $y$ direction:

$$
h(x)=R \cos \left(q_{s} x\right)+h_{0},
$$

where the average $h_{0}=\langle h(x)\rangle$ is taken hereafter to be zero, $h_{0}=0, R$ is the corrugation amplitude and

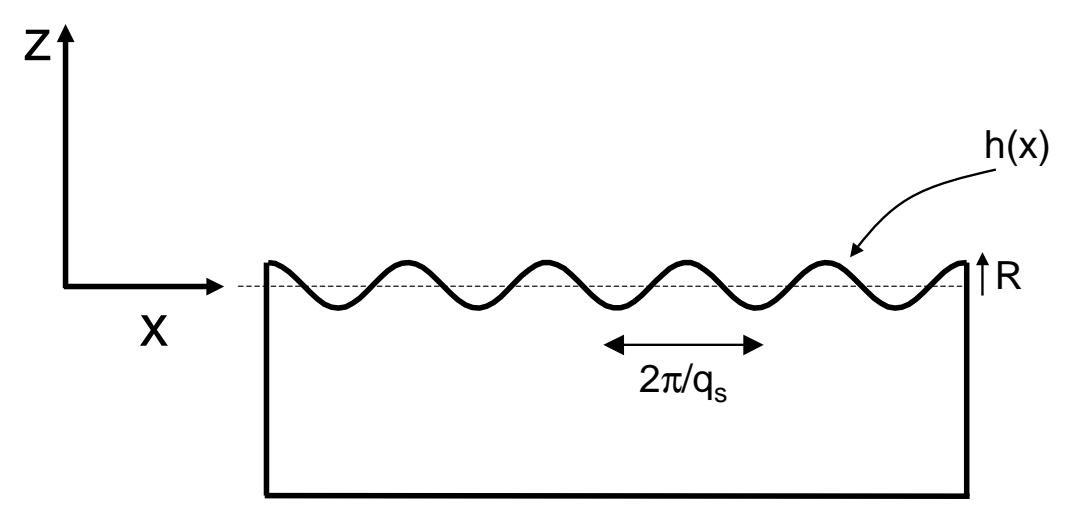

Figure 1: Schematic illustration of the rough confining surface.

$d_{s} \equiv 2 \pi / q_{s}$ is the surface lateral periodicity.

So far we described the quenched corrugated surface geometry. Next we consider the energetics and structure of a lamellar phase in contact with such a surface. We start by examining the order-parameter corresponding to lamellae which are perpendicular to the average surface position:

$$
\phi_{\perp}(\mathbf{r})=\phi_{0} \cos \left(q_{0} x+q_{0} u(x, z)\right)
$$

where $\phi_{0}$ is the amplitude of lamellar concentration variations, $d_{0} \equiv 2 \pi / q_{0}$ is the bulk lamellar spacing, and $u(x, z)$ is a slowly-varying phase describing the deviation from flat lamellae, perpendicular to the surface and given by $\phi_{0} \cos \left(q_{0} x\right)$.

The total free-energy $F$ of the lamellar stack in contact with one surface can be written as a sum of two terms, $F=F_{b}+F_{s}$, where $F_{s}$ is the surface energy and $F_{b}$ is the bulk lamellar contribution. In a lamellar system there are different energy costs associated with bending and compression of the layers. It can be shown that for a slowly varying phase $u(x, z)$, an expansion of the free energy $F_{b}$ to quadratic order in $u$ and its spatial derivatives, can be written in complete analogy with smectic phases of liquid crystals as:

$$
F_{b}=\frac{1}{2} \int\left[K\left(u_{z z}\right)^{2}+B\left(u_{x}\right)^{2}\right] \mathrm{d} V .
$$

In the above equation the integral is over the entire volume, $B$ is the compression modulus and $K$ is the bending modulus. In BCP systems the model holds well in intermediate and strong segregations, where the two copolymer blocks are well separated in different domains and the chains are highly stretched. The elastic moudlii are $K \sim d_{0} \gamma_{\mathrm{AB}}$ and $B \sim \gamma_{\mathrm{AB}} / d_{0}$, where $\gamma_{\mathrm{AB}}$ is the interfacial tension between the $\mathrm{A}$ and $\mathrm{B}$ polymer blocks, and $d_{0}$ is the lamellar periodicity [18. For typical di-BCP such as polystyrene/polymethylmethacrylate (PS/PMMA), where $\gamma_{\mathrm{AB}} \simeq 2 \mathrm{mN} / \mathrm{m}$ and $d_{0} \simeq 50 \mathrm{~nm}$, we estimate their values to be $K \simeq 10^{-11} \mathrm{~J} / \mathrm{m}$ and $B \simeq 4 \cdot 10^{5} \mathrm{~J} / \mathrm{m}^{3}$. For liquid crystals, $K \simeq 2 \cdot 10^{-11} \mathrm{~J} / \mathrm{m}$ and 
$B \simeq 10^{7} \mathrm{~J} / \mathrm{m}^{3}$ [19], whereas for lyotropic (water/surfactant/oil) systems, $K$ is about $1 k_{B} T / d_{0} \simeq 3 \cdot 10^{-30}$ $\mathrm{J} / \mathrm{m}$ and $B \simeq 1 \mathrm{~J} / \mathrm{m}^{3}[20$. Clearly these lyotropic phases are very 'soft' and their elastic modulii have small values.

The penetration length arising from Eq. (3) is

$$
\lambda \equiv(K / B)^{1 / 2}
$$

In lamellar di-BCP this length is proportional to the lamellar spacing, $\lambda \sim d_{0} \simeq 50 \mathrm{~nm}$. In lyotropic systems this distance is very small because of the reduced rigidity.

The second term in the free energy is the surface contribution. Similar to previous works [4, 12, 13, we assume a short-range surface field coupled linearly with the lamellar order-parameter at the surface.

$$
F_{s}=\int \sigma \phi \mathrm{d} S
$$

The parameter $\sigma$ is the surface field and the integral is taken over the entire corrugated surface. The value of $\sigma$ is taken to be a constant throughout the surface, describing a corrugated surface which is chemically homogeneous. A positive (negative) $\sigma$ favors adsorption of negative (positive) $\phi$ at the surface. In the language of an $\mathrm{A} / \mathrm{B}$ di-BCP, this means that $\mathrm{B}$ (or A) monomers are preferentially adsorbed on the surface for $\sigma>0(\sigma<0)$. Assuming small distortions in the order parameter close to the surface, $F_{s}$ can be expanded to first order in $u$

$$
F_{s} \simeq \sigma \phi_{0} \int\left[\cos \left(q_{0} x\right)-q_{0} u \sin \left(q_{0} x\right)\right] \mathrm{d} S
$$

The entire profile $\phi(x, z)$ can now be calculated by using a variational principle on the bulk freeenergy, Eq. (3). In terms of the phase $u(x, z)$, the resulting Euler-Lagrange differential equation away from the corrugated surface is,

$$
\lambda^{2} u_{z z z z}-u_{x x}=0
$$

This variation has to be complemented by a set of rather complex boundary conditions. They are obtained by taking the variation of the full $F_{b}+F_{s}$ on the corrugated surface, $z=h(x)$, defined in Eq. (11),

$$
\begin{aligned}
\frac{\partial f_{b}}{\partial u_{z z}} & =0 \\
\frac{\partial f_{s}}{\partial u}+\left(\frac{\partial f_{b}}{\partial \nabla u}-\frac{\partial}{\partial z} \frac{\partial f_{b}}{\partial u_{z z}} \hat{z}\right) \cdot \hat{n} & =0
\end{aligned}
$$

The unit vector normal to the surface is defined as: $\hat{n}=-\left(q_{s} R \sin q_{s} x, 1\right) / \sqrt{\left(q_{s} R \sin q_{s} x\right)^{2}+1}$, and $f_{b}$ and $f_{s}$ are the integrand of the volume and surface integrals, Eqs. (3) and (6), respectively.

In order to proceed we need to make some further simplifications. Solving the partial differential equation, Eq. (7), with the complex boundary condition, Eqs. (8)-(9) is an extremely difficult task. A further simplification is to assume that $u(x, z)$ is given by the single bulk mode $q_{0}$ in the $x$ direction, while it contains a sum over all possible Fourier modes in the $z$ direction:

$$
u(x, z)=\mathrm{e}^{i q_{0} x} \sum_{k} A_{k} \mathrm{e}^{i k z}+c . c .
$$

This simple sinusoidal form of $u$ along the surface $x$ direction may not fully account for the incommensurability that exists between the lamellar and surface periodicities. Defects and vacancies in the lamellar 
ordering along the surface are not included either. Equations (7) and (10) lead to a selection of specific $k$ modes depending on the bulk mode $q_{0}$ and the penetration length $\lambda$ :

$$
k^{4}+\lambda^{-2} q_{0}^{2}=0
$$

This equation has two different roots, $k_{ \pm}$(and their two complex conjugates)

$$
k_{ \pm}= \pm\left(\frac{q_{0}}{\lambda}\right)^{1 / 2} \cdot \mathrm{e}^{ \pm i \pi / 4}
$$

where $\exp (i \pi / 4)=(1+i) / \sqrt{2}$ is the 4 th root of unity in the complex plane. The first boundary condition, Eq. (8), implies that $A_{k_{+}}=A_{k_{-}}$. Throughout this paper we assume $q_{s} R<q_{0} R \ll 1$, whence

it is valid to approximate $\sqrt{\left(q_{s} R \sin q_{s} x\right)^{2}+1} \simeq 1$ and $\exp \left(i k R \cos \left(q_{s} x\right)\right) \simeq 1$. The second boundary condition [Eq.(91)] then gives

$$
-\sigma \phi_{0} q_{0} \sin q_{0} x-i B q_{s} R \sin q_{s} x \cdot \sum_{k} q_{0} A_{k} \mathrm{e}^{i\left(q_{0} x+k z\right)}-i K \cdot \sum_{k} k^{3} A_{k} \mathrm{e}^{i\left(q_{0} x+k z\right)}=0
$$

The $z$-dependent term in the exponentials is neglected as can be justified for $q_{0} R \ll 1$. The second term (proportional to $B$ ) can also be neglected for $q_{s} R \ll 1$, leading to the final expression of the lamellar order parameter:

$$
\begin{aligned}
u & =A_{0} \mathrm{e}^{i\left(q_{0} x+k_{+} z\right)}+A_{0} \mathrm{e}^{i\left(q_{0} x+k_{-} z\right)}+c . c . \\
A_{0} & =\frac{\sigma \phi_{0} q_{0}}{2 K\left(k_{+}^{3}+k_{-}^{3}\right)}
\end{aligned}
$$

where $A_{q_{0}}$ is denoted $A_{0}$ in order to simplify the notation. The full order-parameter expression is obtained by substituting $u$ from above in Eq. (2). The decay length of lamellar undulations is proportional to the lamellar spacing, $1 / k_{ \pm} \sim\left(\lambda / q_{0}\right)^{1 / 2} \sim d_{0}$.

The perpendicular lamellar stack on a rough surface is shown in Fig. 2. In Fig. 2(a) the lamellar periodicity is half of the surface one. The surface is attractive to the component marked in dark shades, causing them to expand in its vicinity. Light lamellae are in turn contracted close to the surface; this behavior is seen in part 2(c) too, where the surface periodicity matches the bulk lamellar one. Clearly, the curvature of the lamellae adjusts to the surface one in order to achieve the best compromise between elastic deformation and surface coverage.

\section{Parallel Layers on Corrugated Surface}

We now turn to describe a parallel lamellar stack in contact with the same sinusoidally corrugated surface. Our derivation is related to previous treatments [7] 21, 22, but the boundary conditions are handled differently by introducing the same type of surface term in the free energy $F_{s}$, as in the previous section. The lamellae can be described along the same lines as for the perpendicular case but keeping in mind the important points where the stack orientation affects the free energy:

$$
\phi_{\|}(\mathbf{r})=-\phi_{0} \cos \left(q_{0} z+q_{0} u(x, z)\right)
$$

The perfect parallel layers, $\phi_{\|}(\mathbf{r})=-\phi_{q} \cos q_{0} z$ are recovered far from the surface (where $u=0$ ). The bulk lamellar phase free-energy is obtained simply by interchanging the roles of $x$ and $z$ axes in the free energy of Eq. (3): $x \leftrightarrow z$

$$
F_{b}=\frac{1}{2} \int\left[K\left(u_{x x}\right)^{2}+B\left(u_{z}\right)^{2}\right] \mathrm{d} V
$$




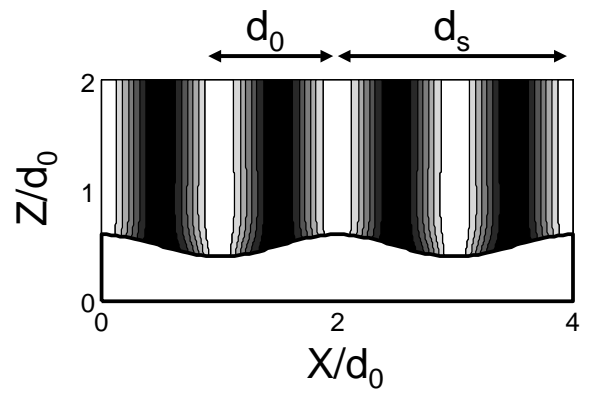

(a)

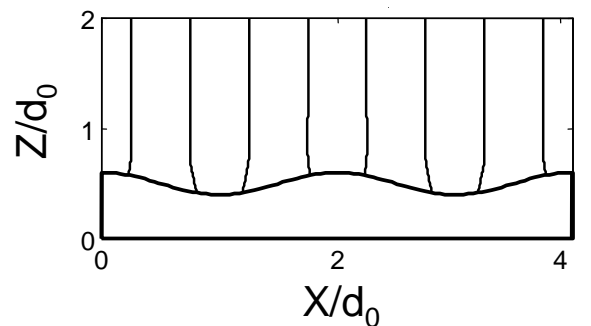

(b)

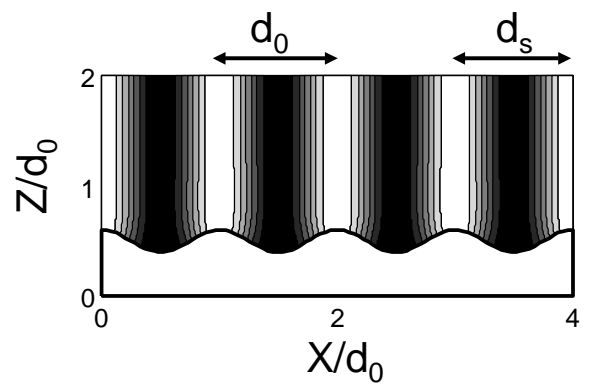

(c)

Figure 2: Perpendicular lamellar layers in contact with a sinusoidal surface, as obtained from Eqs. (2) and (14). All length are scaled by $d_{0}$, the lamellar bulk periodicity, and the origin of the $z$ axis is shifted by $d_{0} / 2$ for clarity purposes. (a) The lamellar periodicity $d_{0}$ is chosen to be half of the surface one, $d_{0}=d_{s} / 2$. Different grey shades correspond to different values of the order parameter $\phi$ of Eq. (2), where dark and white correspond to negative and positive $\phi$, respectively. (b) Inter-material dividing surface corresponding to lines where $\phi=0$ in part (a). (c) The same as in (a), but the lamellar periodicity equals the surface one, $d_{s}=d_{0}$. The parameters used are: $B=4, K=B /\left(4 q_{0}^{2}\right)$ and $\sigma=\sqrt{B K} / 4$.

As for the case of perpendicular lamellae, the surface energy, Eq. (5), can be expanded in small $u$ as follows

$$
F_{s} \simeq \sigma \phi_{0} \int\left[q_{0} u \sin \left(q_{0} z\right)-\cos \left(q_{0} z\right)\right] \mathrm{d} S
$$

The governing equation for $u$ is obtained from a variation principle applied to Eq. (16),,$\delta F_{b} / \delta u=0$

$$
\lambda^{2} u_{x x x x}-u_{z z}=0
$$

with the boundary condition obtained from a variation of $F_{b}+F_{s}$ on the $z=h(x)$ corrugated surface.

$$
\frac{\partial f_{s}}{\partial u}+\left(\frac{\partial f}{\partial \nabla u}-\frac{\partial}{\partial x} \frac{\partial f}{\partial u_{x x}} \hat{x}\right) \cdot \hat{n}=0
$$

Writing $u$ as $u(x, z)=A_{0} \mathrm{e}^{i \alpha z} \cos q_{s} x$ and using Eq. (18) we find that $\alpha=i \lambda q_{s}^{2}$. At this point it should be emphasized that when the surface periodicity is larger than the lamellar periodicity $\left(q_{s}<q_{0}\right)$, 
the decay length $1 / \alpha \sim q_{0} / q_{s}^{2}$ for the parallel stack is much larger than the decay length $1 / k_{ \pm} \sim 1 / q_{0}$ in the perpendicular case. This, in turn, means that for fixed $R$ the elastic deformation gives preference to perpendicular ordering.

As in the case of perpendicular lamellae, we use $q_{s} R<q_{0} R \ll 1$ to approximate $\sqrt{\left(q_{s} R \sin q_{s} x\right)^{2}+1} \simeq 1$ and obtain from the boundary condition, Eq. (19),

$$
\sigma \phi_{0} q_{0}^{2} R+B \lambda q_{s}^{2} A_{0}=0
$$

yielding the order-parameter of parallel lamellae

$$
\begin{aligned}
u(x, z) & =A_{0} \mathrm{e}^{i \alpha z} \cos q_{s} x \\
A_{0} & =-\frac{\sigma \phi_{0} q_{0}^{2} R}{B \lambda q_{s}^{2}}=-R \frac{\sigma \phi_{0}}{\sqrt{B K}}\left(\frac{q_{0}}{q_{s}}\right)^{2}
\end{aligned}
$$

The parallel layering given by Eq. (21) is plotted in Fig. 3. In 3(a) [as in Fig. 2(a)] the surface periodicity is twice larger than the lamellar one, and in 3(b) the two periodicities are equal. In the former case, dark regions (negative $\phi$ ) appear close to the wall, the lamellae are able to closely follow the surface contour and distortions are long-range. In the latter case the surface topography changes too quickly for the lamellae to follow and the lamellae lie almost perfectly flat. Distortions of the stack, in this case, can be seen only in the very close vicinity of the surface.

\section{Phase Diagram}

The elastic energy of the lamellar phase in the two orientations is obtained by substitution of Eq. (14) in Eqs. (3) and (5) (perpendicular lamellae) and Eq. (21) in Eqs. (16) and (5) (parallel lamellae). Depending on the system parameters $R, q_{s}, q_{0}$ and $\sigma$, the minimum of the free energy is obtained for either one of the two orientations.

Before we proceed in presenting the corresponding phase diagram, we note that our derivation is not valid over the entire $R, q_{s}, q_{0}$ and $\sigma$ parameter space. The assumption of small distortions, $q_{0} u \ll 1$, together with Eq. (21) implies that $R \sigma \phi_{0} q_{0}^{3} q_{s}^{-2} \ll \sqrt{B K}$. This can be rewritten as $q_{s} R \gg$ $\left(q_{0} R\right)^{3 / 2}\left(\sigma \phi_{0} / \sqrt{B K}\right)^{1 / 2}$. Combining these two inequalities, we obtain that the limits of validity of our derivation are given by

$$
1 \gg q_{0} R>q_{s} R \gg\left(q_{0} R\right)^{3 / 2}\left(\frac{\sigma \phi_{0}}{\sqrt{B K}}\right)^{1 / 2}
$$

Cast in different terms this can be written as

$$
\left(\frac{\sigma \phi_{0}}{\sqrt{B K}}\right)^{1 / 2}\left(q_{0} R\right)^{1 / 2} \ll \frac{q_{s}}{q_{0}}<1
$$

Before presenting the calculated phase diagram, it is of use to present a rough estimation of the parallel and perpendicular free-energies. Disregarding numerical prefactors, the bulk free energy of the perpendicular state is

$$
\begin{aligned}
\frac{F_{\perp}}{S} & \sim A_{0}^{2}\left(B q_{0}^{2}+K k_{ \pm}^{4}\right) \cdot \frac{1}{\operatorname{Im}\left(k_{ \pm}\right)} \\
& =\frac{\sigma^{2} \phi_{0}^{2}}{K} \frac{1}{q_{0}}
\end{aligned}
$$




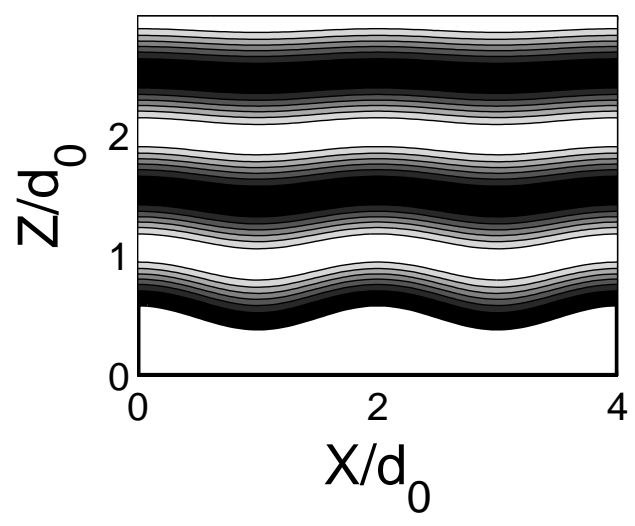

(a)

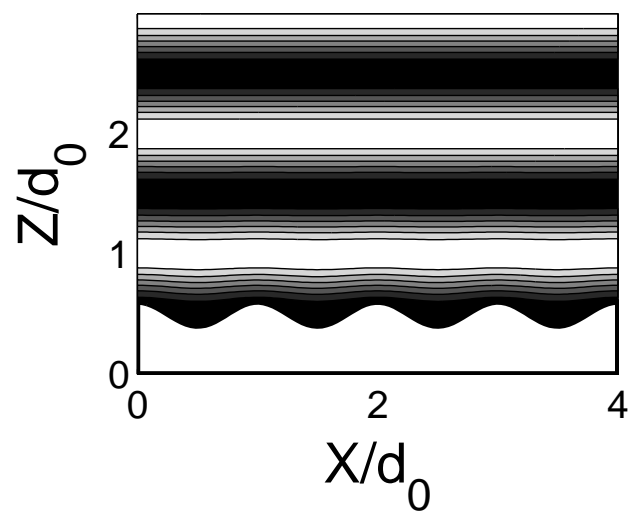

(b)

Figure 3: Parallel lamellar layers in contact with a corrugated surface, calculated from the free energy minimization of Sec. 3. (a) The lamellar periodicity is half of the surface one $d_{0}=d_{s} / 2$, and the lamellae follow the surface topography. (b) The two periodicities are equal, $d_{s}=d_{0}$, and the lamellae are unable to follow the surface height variations. Parameters here are the same as in Fig. 2.

where $S$ is the surface area and $A_{0}$ is taken from Eq. (14). At distances from the surface greater than $1 / \operatorname{Im}\left(k_{ \pm}\right)$, the distortion $u$ is negligible, and the factor $1 / \operatorname{Im}\left(k_{ \pm}\right)$represents the effective volume to area ratio of the integration.

The free energy of the parallel state is similarly estimated to be

$$
\begin{aligned}
\frac{F_{\|}}{S} & \sim A_{0}^{2}\left(B \alpha^{2}+K q_{s}^{4}\right) \cdot \frac{1}{\operatorname{Im}(\alpha)} \\
& =\frac{\sigma^{2} \phi_{0}^{2}}{K} \frac{1}{q_{0}}\left(\frac{q_{0}}{q_{s}}\right)^{2}\left(q_{0} R\right)^{2}
\end{aligned}
$$

Here $A_{0}$ is taken from Eq. (21) and the effective volume to area ratio is $1 / \operatorname{Im}(\alpha)$.

The difference between the two free energies is roughly proportional to

$$
F_{\|}-F_{\perp} \propto\left(\frac{q_{0}}{q_{s}}\right)^{2}\left(q_{0} R\right)^{2}-1
$$

Eq. (26) describes qualitatively the system behavior. If $q_{0}$ is fixed while $R$ and $q_{s}$ can vary, it directly follows that for $q_{0} R>q_{s} / q_{0}$ the perpendicular state is favored, while for $q_{0} R<q_{s} / q_{0}$ the parallel state is favored. Similar relations hold when $q_{s}$ is fixed and $R$ and $q_{0}$ are allowed to vary, or when $R$ is fixed but $q_{s}$ and $q_{0}$ can vary. 


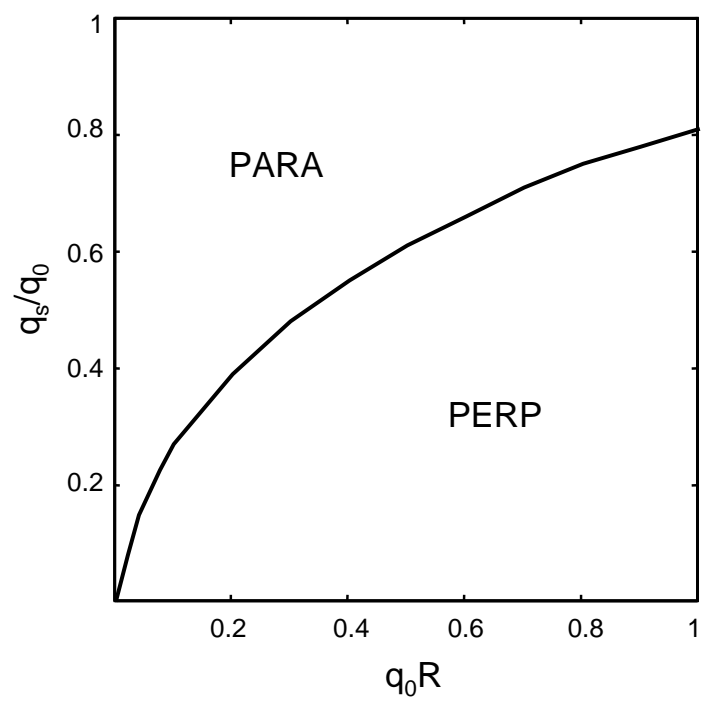

Figure 4: Phase diagram in terms of $q_{s}$ and $R$ with fixed surface field $\sigma$ and lamellar periodicity $2 \pi / q_{0}$. For fixed value of $q_{s}$ (scaled by $q_{0}$ ), a horizontal scan of increasing $R$ (scaled by $1 / q_{0}$ ) will increase the elastic penalty of parallel layers, and favors perpendicular ordering. On the other hand, a vertical scan of increasing $q_{s}$ (while keeping $R$ constant) limits the deformations in the parallel state to the surface, and favors parallel ordering. $\sigma=2 \sqrt{B K}$ and all other parameters are the same as in Figs. 2 and 3 .

The above simplified approach describes qualitatively the system behavior and may be used as a 'rule of thumb'. Furthermore, the surface energies and the correct numerical prefactors can be taken into account as well. Figure 4 shows the phase diagram in the $\left(q_{s}, R\right)$ plane, for fixed $q_{0}$ and $\sigma$. Not the entire shown phase diagram is within the range of validity discussed above, Eq. (23). For small values of $q_{s}$, the perpendicular layering is favored, because of the long-ranged elastic strain pertaining in parallel lamellae as compared to perpendicular ones. As $q_{s}$ increases, the strain in the parallel state becomes more restricted to the vicinity of the surface, until, eventually, the parallel state becomes more stable. At this transition point, the energetic gain of having a commensurate layer close to the surface overcomes the loss of elastic energy deformation.

Different conclusion can be drawn for small values of surface amplitude $R$, which generally induce a parallel state. Keeping $q_{s}$ fixed and gradually increasing $R$ means that the elastic energy of deforming parallel lamellae increases, while the surface interaction stays constant. Therefore, at a certain threshold value of $R$ there is a transition from parallel to perpendicular ordering. For larger $q_{s}$ values this critical $R$ value increases as well.

Figure 5 is a phase diagram in the $\left(R, q_{0}\right)$ plane, with $q_{s}$ kept fixed. For a given BCP chain length (fixed $q_{0}$ ), increase of $R$ will also increase the elastic energy of deforming parallel lamellae, and promotes perpendicular layering. On the other hand, keeping $R$ fixed and decreasing $q_{0}$ (so that it becomes comparable to $q_{s}$ ) decreases the range of parallel deformation; thus, favoring the parallel state.

\section{Discussion}

Lamellar stacks of either liquid crystals (smectics), short chains amphiphiles or long-chain BCP undergo deformation as they try to adjust to the presence of a rough (corrugated) surface. The amplitude and spatial extent of inplane and out-of-plane deformations are different and the preference for parallel or 


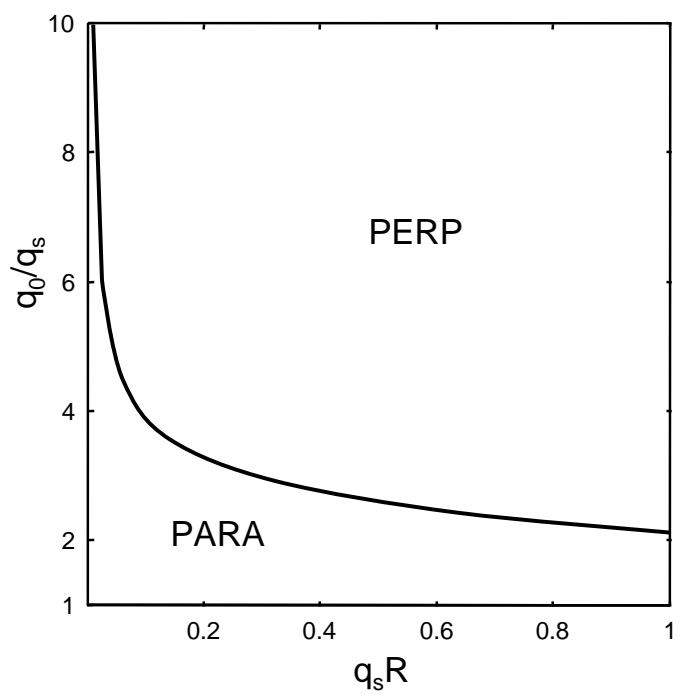

Figure 5: Phase-diagram in the $\left(R, q_{0}\right)$ plane. The surface field $\sigma$ and surface periodicity $2 \pi / q_{s}$ are kept constant. Horizontal scans of increasing $R$ (scaled by $1 / q_{s}$ ) while keeping $q_{0}$ fixed, increases the elastic penalty of parallel layers and favors perpendicular ordering. Same is true for vertical scans of increasing $q_{0}$ (scaled by $q_{s}$ ) with fixed $R . \sigma=0.2 \sqrt{B K}$ and other parameters are the same as in Figs. 2 and 3.

perpendicular orientations depends on specific bulk and surface parameters. Which morphology prevails depends on the surface periodicity $d_{s}=2 \pi / q_{s}$, surface amplitude $R$, lamellar periodicity $d_{0}=2 \pi / q_{0}$, as well as the surface field strength $\sigma$.

For lamellae oriented perpendicular to the surface we use a model that indicates how the stack deformation propagates from the surface into the semi-infinite bulk. Parallel lamellae are studied using a modification of a model used previously for smectics phase. In both cases small surface corrugation is assumed such that we can use the limits: $q_{s} R \ll 1, q_{0} R \ll 1$. Our study is complementary to a numerical study by Podariu and Chakrabarti 23] who studied in detail extremely thin films (thickness comparable or smaller than the lamellar thickness) of a lamellar stack.

The system behavior in terms of the parameters $R$ and $q_{s}$ is given in the phase diagram of Fig. 4 . For fixed surface periodicity $2 \pi / q_{s}$, increase in the surface amplitude $R$ leads to preference of perpendicular lamellae. On the other hand, keeping $R$ fixed and increasing $q_{s}$ leads to preference of parallel lamellae. This is a consequence of the diminished decay length of surface-induced undulations. When the undulations of parallel lamellae are restricted to the surface vicinity, the energy penalty of the elastic defect can be small. Thus, the phase transition between these two states is described by a line in the two-dimensional phase diagram. Quite generally, as the surface interaction parameter $\sigma$ is increased, this line moves towards the $R$ axis in such a way that the state of parallel lamellae occupies a larger region in the phase diagram.

The transition from parallel to perpendicular orientation as function of $R$ and $q_{0}$ is given in Fig. 5 . Increase of $R$ deforms the parallel layers and generally promotes perpendicular ordering. On the other hand, increase of $q_{0}$ while keeping $R$ and $q_{s}$ fixed, implies a reduced range of parallel deformations and yield a preference for parallel lamellae.

Rough surface can be used to obtain morphologies that are usually controlled by chemical means: not only the transition between parallel and perpendicular lamellae, but also tilted lamellar morphologies. Indeed, in the limit of very small lamellar periodicity (large $q_{0}$ ) and strong interfacial interactions $\sigma$, 
lamellae will appear locally perpendicular to the surface, and therefore tilt when the surface is not horizontal. Similar tilted lamellae have been predicted for a BCP melt confined by chemically patterned surfaces [3, 4], as the system tries to match the lamellae with the stripes.

The present work is motivated by and is of direct relevance to recent experiments of Hashimoto and co-workers on PS/PMMA symmetric di-BCP on rough surfaces [17]. In the experiments, perpendicular orientation was observed for system parameters estimated to be $q_{s} \simeq 0.04 \mathrm{~nm}^{-1}, q_{0} \simeq 0.33 \mathrm{~nm}^{-1}$ and $R \simeq 7.5 \mathrm{~nm}$. This set of data is in complete accord with the scaling formula, Eq. (26), because $q_{0}^{4} R^{2} / q_{s}^{2}-1$ is large and positive. This also can be confirmed by the phase diagrams presented in Figs. 4 and 5 .

For different surfaces and BCP films reported in Ref. [17], $q_{s} \simeq 0.018 \mathrm{~nm}^{-1}, q_{0} \simeq 0.19 \mathrm{~nm}^{-1}$ and $R \simeq 2.7 \mathrm{~nm}$, and the film orients itself in parallel layers. The scaling formula, Eq. (26) yields here a small positive number, implying weak preference for perpendicular layers. The phase diagram also show marginal behavior, possibly preferential for perpendicular layers. This discrepancy can have several (yet unknown) origins. First, the exact value of the surface interaction parameter $\sigma$ is not known from experiments, and it may be different than the values chosen by us in Figs. 4 and 5 . Furthermore, we employed several approximations in our calculations ignoring, for example, possible vacancies in the perpendicular lamellae and the temperature dependence of the elastic modulii. We also assumed that the system is semi-infinite in the $z$-direction, while in experiments the BCP film thickness is finite. It may be that the $\mathrm{BCP} /$ air free surface can induce islands or other types of surface-induced defects that alter the simple picture employed by us. The free-energy model holds for BCPs in intermediate and strong segregations, but is less adequate for weaker segregations, as is possibly the case in the experiments. Lastly, our theory can be improved by taking into account more realistic surface roughness, instead of a single surface $q$-mode.

We hope that additional and detailed experiments of lamellar systems in contact with well characterized rough surfaces will shed more light on this problem, and will further motivate theoretical studies of these intriguing systems.

Acknowledgements. We thank T. Hashimoto and E. Sivaniah for many discussions and correspondence, and for sharing with us their experimental results prior to publication. We thank M. Cloitre, J.-B. Fournier, F. Tournilhac and L. Leibler for useful discussions and comments. DA wishes to acknowledge support from the Israel Science Foundation under grant no. 210/02, and the Alexander von Humboldt Foundation, while YT thanks the Chateaubriand fellowship program. 


\section{References}

[1] Lambooy, P.; Russell, T. P.; Kellogg, G. J.; Mays, A. M.; Gallagher, P. D.; Satija, S. K. Phys. Rev. Lett. 1994, 72, 2899.

[2] Turner, M. S. Phys. Rev. Lett. 1992, 69, 1788.

[3] Petera, D; Muthukumar, M. J. Chem. Phys. 1998, 109, 5101.

[4] Tsori, Y.; Andelman, D. J. Chem. Phys. 2001, 115, 1970.

[5] Patel, S. S.; Larson, R. G.; Winey, K. I.; Watanabe, H. Macromolecules 1995, 28, 4313. Koppi, K. A.; Tirrell, M.; Bates, F. Phys. Rev. Lett 1993, 70, 1449. Riise, B. L.; Fredrickson, G. H.; Larson, R. G.; Pearson, D. S. Macromolecules 1995, 28, 7653.

[6] Fréedericksz, V.; Zolina, V. Trans. Faraday Soc. 1933, 29, 919.

[7] de Gennes, P. G.; Prost, J. The Physics of Liquid Crystals, 2nd Edition (Oxford University Press, Oxford, 1993).

[8] Amundson, K.; Helfand, E.; Quan, X; Smith, S. D. Macromolecules 1993, 26, 2698.

[9] Amundson, K.; Helfand, E.; Quan, X.; Hudson, S. D.; Smith, S. D. Macromolecules 1994, $27,6559$.

[10] Böker, A.; Knoll, A.; Elbs, H.; Abetz, V.; Müller, A. H. E.; Krausch, G. Macromolecules 2002, 35, 1319.

[11] Thurn-Albrecht, T.; Schotter, J.; Kästle, G. A.; Emley, N.; Shibauchi, T.; Krusin-Elbaum, L.; Guarini, K.; Black, C. T.; Tuominen, M. T.; Russell, T. P. Science 2000, 290, 2126.

[12] Pereira, G. G. ; Williams, D. R. M. Macromolecules 1999, 32, 8115. Ashok, B.; Muthukumar, M.; Russell, T. P. J. Chem. Phys. 2001, 115, 1559.

[13] Tsori, Y.; Andelman, D. Macromolecules 2002, 35, 5161.

[14] Tsori, Y.; Tournilhac, F.; Andelman, D.; Leibler, L. Phys. Rev. Lett. 2003, 90, 145504.

[15] Tsori, Y.; Tournilhac, F.; Leibler, L. Submitted to Macromolecules (2003).

[16] Morariu, M. D.; Voicu, N. E.; Schäffer, E.; Lin, Z.; Russell, T. P.; Steiner, U. Nature Materials 2003, 2, 48 .

[17] Sivaniah, E.; Hayashi, Y.; Iino, M.; Fukunaga, K.; Hashimoto, T. Submitted to Macromolecules, (2002).

[18] Turner, M. S.; Maloum, M.; Ausserré, D.; Joanny, J. -F.; Kunz, M. J. Phys. II France 1994, 4, 689.

[19] Martinoty, P.; Gallani, J. L.; Collin, D. Phys. Rev. Lett. 1998, 81, 144.

[20] Bougket, G.; Ligoure, C. Eur. Phys. J. B 1999, 9, 137.

[21] Turner, M. S.; Joanny, J.-F. Macromolecules 1992, 25, 6681.

[22] Li, Z.; Qu, S.; Rafailovich, M. H.; Sokolov, J.; Tolan, M.; Turner, M. S.; Wang, J.; Schwarz, S. A.; Lorenz, H.; Kotthaus, J. P. Macromolecules 1997, 30, 8410.

[23] Podariu, L.; Chakrabarti, A. J. Chem. Phys. 2000, 113, 6423. 